\title{
A Systematic Review of the Efficacy of Bioactive Compounds in Cardiovascular Disease: Carbohydrates, Active Lipids and Nitrogen Compounds
}

\author{
Belen Pastor-Villaescusa Oscar D. Rangel-Huerta Concepcion M. Aguilera \\ Angel Gil \\ Department of Biochemistry and Molecular Biology II, Centre of Biomedical Research, Institute of Nutrition and \\ Food Technology 'José Mataix', University of Granada, Granada, Spain
}

\section{Key Words}

Bioactive food compounds . Cardiovascular diseases .

Carbohydrates · Fatty acids · Nitrogen compounds

\begin{abstract}
Background/Aims: The prevalence of cardiovascular diseases (CVD) is rising and it is the prime cause of death in all developed countries. Bioactive compounds (BACs) can play a role in CVD prevention and treatment. To examine the scientific evidence supporting BACs groups' efficacy in CVD prevention and treatment, we conducted a systematized review. Methods: All available information on Medline, LILACS and EMBASE; all randomized controlled trials (RCTs) with prospective, parallel or crossover designs in humans in which the BACs effect was compared with that of placebo/ control. Vascular homeostasis, blood pressure, endothelial function, oxidative stress and inflammatory biomarkers were considered primary outcomes. Results: We selected 26 articles, verifying their quality based on the Scottish Intercollegiate Guidelines Network, establishing diverse quality levels of scientific evidence according to the design and bias risk of a study. Grades of recommendation were included,
\end{abstract}

depending on the evidence strength of antecedents. Conclusions: Evidence shows that certain BACs' derivative from active lipids and nitrogen compounds, mainly from horse chestnut seed extract, sterol plants, allium derivatives, and certain doses of beta-glucans, can be helpful in decreasing the prevalence of CVD risk factors. However, further rigorous evidence is necessary to support and prove BACs' effect on CVD prevention and treatment.

() 2015 S. Karger AG, Basel

\section{Introduction}

The prevalence of cardiovascular disease (CVD) is increasing by the day; it is the prime cause of death in all developed countries [1] and one of the most important for developing countries [2]. There are some risk factors that cannot be changed such as family history, ethnicity and age. However, detection and control of modifiable factors such as, blood pressure (BP), high cholesterol,

B.P.-V. and O.D.R.-H. contributed equally to the present work.

\section{KARGER 125}

(c) 2015 S. Karger AG, Base

$0250-6807 / 15 / 0663-0168 \$ 39.50 / 0$

E-Mail karger@karger.com

www.karger.com/anm
Department of Biochemistry and Molecular Biology II, Institute of Nutrition and Food Technology 'José Mataix', Centre of Biomedical Research, University of Granada Avenida del Conocimiento s/n, ES-18006 Granada (Spain)

E-Mail agil@ugr.es 
obesity, type 2 diabetes (T2D) or unhealthy diets can help to prevent intermediate risk CVD processes like inflammation or oxidative stress. Thus, the primary prevention of CVD by identifying and treating at-risk individuals remains a major public-health priority. A healthy life style is the main preemptive approach $[3,4]$.

Dietary habits are quite different around the world; nevertheless, certain consumption patterns are common worldwide like inclusion of fruits and vegetables or products like cocoa, coffee or condiments is a merging point. Bioactive compounds (BACs) are 'extra nutritional' constituents that are present in small quantities in plant products and lipid-rich foods [5]. The growing body of scientific evidence indicates that certain BACs play a beneficial role in CVD prevention $[5,6]$. BACs oral supplements taken along with the usual diet can increase the intake of ingredients reputed to have clinical benefit. These supplements are, usually, an addition to a healthy diet, and not a substitution to conventional food or the sole item of a meal [7].

Putative beneficial biological effects such as antilipidemic, antihypertensive, anti-glycaemic, antithrombotic and anti-atherogenic effects are attributed to BACs. In this study, the main goal was to examine the scientific evidence of the role of BACs in the prevention and treatment of CVD by a systematized review of randomized clinical trials (RCTs). The vegetal BACs considered in this review were those derived from carbohydrates, active lipids and organosulfur compounds.

Epidemiologic studies have suggested that dietary fibres such as $\beta$-glucan, a non-starch viscous polysaccharide derived from oat, are known to exhibit hypercholesterolemic effects, furthermore protecting against $\mathrm{CHD}$ [8].

Concerning active lipids, we can find that the conjugated linoleic acids (CLAs) are a group of minor fatty acids from ruminant origin, which has long been recognized as a body fat-lowering agent. Dairy food products are rich in CLA, which is considered an important component due to its potential health benefits such as anticarcinogenic, anti-atherogenic, antidiabetic and anti-adipogenic properties [9]. Additionally, the terpenes compounds are constituted by monoterpenes, which are naturally occurring isoprenoids found in the essential oils of citrus fruits, cherries, mint, and herbs [10]. Diterpenoids form a large class of plant-derived secondary metabolites that possess a wide spectrum of important biological activities; an example is the extract from seeds and bark of horse chestnut (Aesculus hippocastanum L.) that is used as a herbal medicine to treat chronic venous insuf- ficiency [11]. Moreover, plant sterols (or phytosterols) are natural constituents of cell membrane of plants. Their role in plants is similar to that of cholesterol in humans [12]. It has been known since the 1950s, that plant sterols inhibit cholesterol absorption, and since the 1980s that plant stanols inhibit cholesterol absorption [13].

Other compounds included are those derived from the Allium sativum, commonly known as garlic, which is a plant of the family Alliaceae and is widely consumed as a spice in Korea. Garlic contains high levels of organosulfur compounds and flavonoids as well as a variety of compounds that function synergistically to provide various health benefits [14].

Along with tea, coffee is the most common hot drink prevalent in many cultures. Caffeine (1,3,7-trimethylxanthine), a purine alkaloid contained in the seeds of the coffee plant, represents the most abundant component in coffee, and its potential effects on health still represent a controversial issue.

We thoroughly examined the effects of BACs on BP, lipids profile (triacylglycerol, cholesterol, high and low density lipoproteins), carbohydrate $(\mathrm{CHO})$ metabolism (glucose, insulin, and insulin resistance), oxidative stress, inflammation and endothelial function (EF). Furthermore, we gave a recommendation for its consumption based on the evidence grade according to Scottish Intercollegiate Guidelines Network (SIGN) [15].

\section{Methods}

We developed a literature search in Medline by PubMed (U.S. National Library of Medicine and the NIH), just as in LILACS and EMBASE, including publications in English, Spanish and Portuguese until December 2014. Studies eligible for this review included randomized controlled trials (RCTs) in healthy and unhealthy adults, with prospective, parallel or crossover designs, with full text, and whose primary outcomes were vascular homeostasis, $\mathrm{BP}$, oxidative stress and/or inflammatory biomarkers; we excluded those studies with a cohort, ecological or case-control design, which analyzed whether a drug or BACs were combined with other compounds. However, there was no restriction on the publication type or sample size.

\section{Search Equation}

Due to the diversity of their chemical structures (fig. 1), BACs can present different effects in CVD; consequently, in this section, we included all those related to carbohydrates, active lipids and nitrogen compounds. We included different keywords for the search, which are considered bioactive compounds, including active lipids (linoleic acids, conjugated (CLAs), fatty acids, hydro fatty acids, fatty acids esters, alpha-pinene) and carbohydrates (beta-glucans); terpenes (monoterpenes: iridoids, menthol, norbornanes, pyrethrins, thymol; diterpenes: aonitine, aphidicolin, atractyloside, 


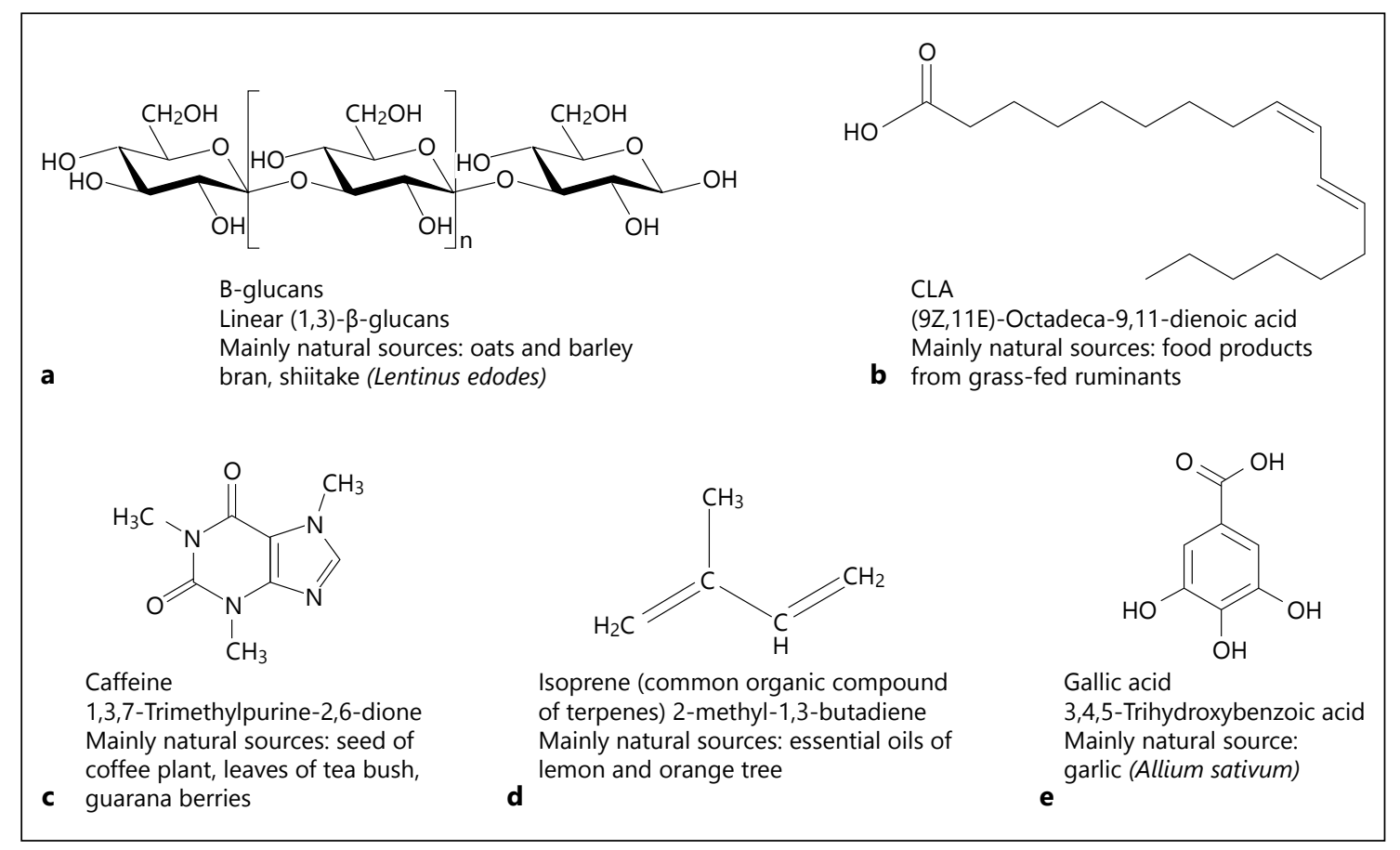

Fig. 1. Chemical diversity of BACs in nature; examples of carbohydrates ( $\beta$-glucan) (a), active lipids (CLAs) (b) and nitrogen compounds: xanthine derivatives $(\mathbf{c})$, terpenes $(\mathbf{d})$, allium compounds $(\mathbf{e})$.

colforsin, abietane, clerodane, kaurane, ginkgolides, phorbols, phytanic acid, phytol, ryanodine, taxoids; triterpenes: cucurbitacins, lanosterol, limonins, pentacyclic triterpenes, quassins, sapogenins; polyterpenoids: phytosterols, ellagic acid, squalene); nitrogen compounds (caffeine, allium, glucosinolates, xanthine, pseudoephedrine, theobromine, theophylline, ephedra); alkaloids (betalains, indole alkaloids, physostigmine, secologanintryptamine alkaloids, staurosporine, peptide alkaloids). We combined the MeSH term 'cardiovascular diseases' with each bioactive compound as a $\mathrm{MeSH}$ major topic, together with NOT 'review' [Publication Type] in PubMed. However, equations in Spanish language were used when the search was carried out in LILACS, that is, (tw:(ácido linoleico)) AND (tw:(enfermedad cardiovascular)) AND NOT (tw:(revisión)). When consulting the database EMBASE, the equations were elaborated as 'catechols'/mj AND 'cardiovascular diseases'/mj NOT 'review'. Articles published before 1990 were discarded because they were not relevant any more.

\section{Selection and Evaluation}

First, both titles and abstracts were identified independently by two reviewers, for discarding those that did not fit with the language, date, subject matter, design and outcomes established. Then, full-text publications were classified based on pathologies according to outcomes analyzed in each study.

Moreover, RCTs were finally selected if they obtained a score between 3 and 5 according to the JADAD scale [16]. Such method pretends to minimize bias for RCTs, ensuring certain quality in the evidence; it took into account if they were randomized, blind and provided detailed information about patients.
Furthermore, we verified the quality of selected articles by the Scottish Intercollegiate Guidelines Network (SIGN) [15]. Diverse quality levels of scientific evidence are established according to two features: the design and bias risk of a study. The levels are from $1++$, when the information is considered high quality, to 4 when the information is considered very low quality. Signs are used to reporting with reference to compliance degree of key criteria associated with potential bias $(1++, 1+, 1-, 2++$, $2+, 2-, 3$, and 4). Additionally, we included a grade of recommendation, based on the evidence strength of the antecedents, whose levels are A, B, C, D, 'A' being highly recommended and ' $\mathrm{D}$ ' not recommended. These grades of recommendation by SIGN guidelines are equivalent to those designated by $\mathrm{FAO} / \mathrm{WHO}$, as evidence criteria: convincing, probable, possible and insufficient [17].

\section{Results}

In total, 760 RCTs were found using the equations proposed in the different databases (EMBASE, LILACS and PubMed). We exclude 715 to obvious irrelevance leaving in 45 full papers to read (fig. 2). Finally, after reading the full paper and evaluating it using the JADAD scale, we selected 26 articles for the final review and they are included in tables 1 and 2 . 
Fig. 2. Review flow diagram of the system-

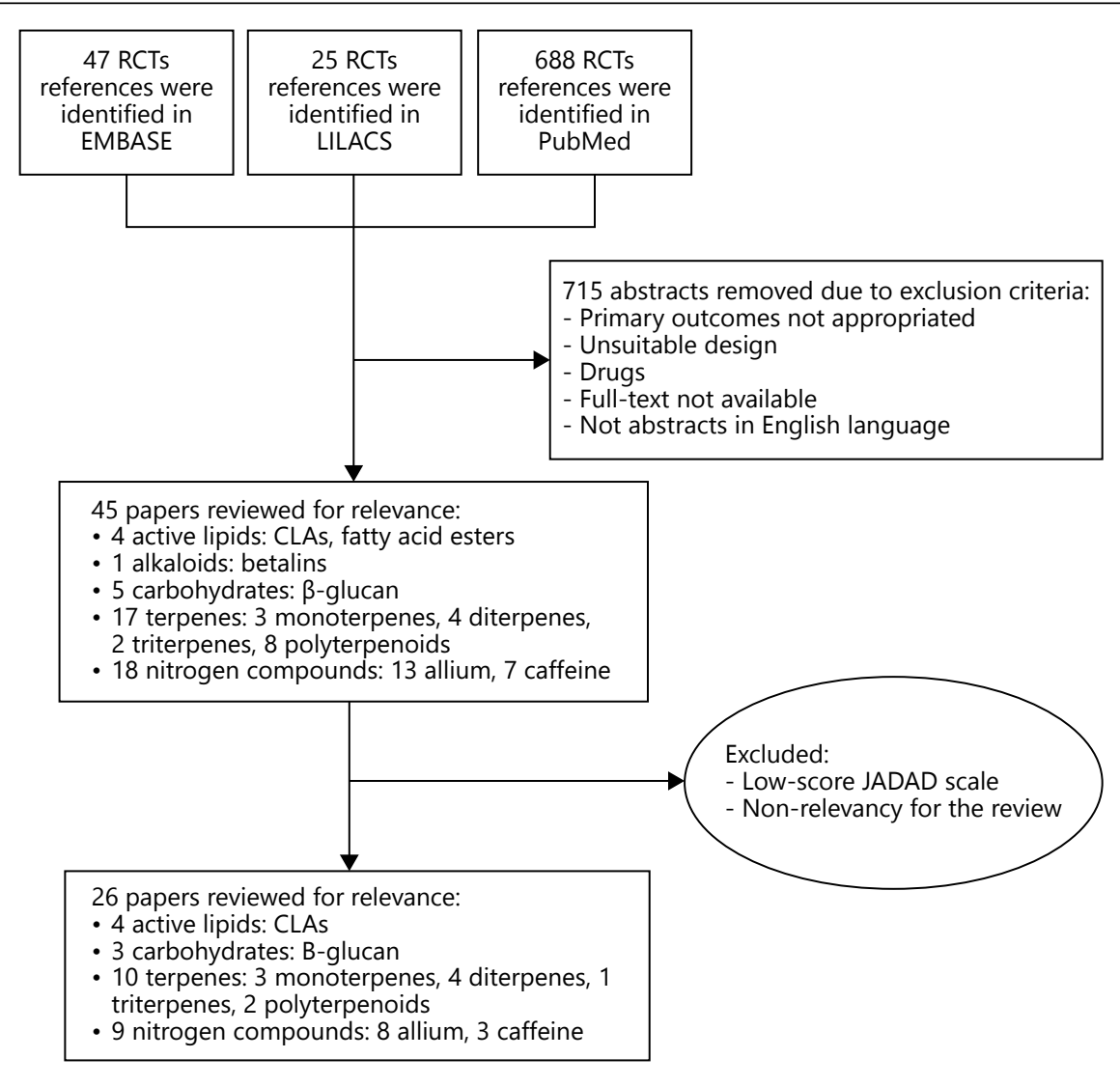
atic review.

\section{Carbohydrates}

Three articles of carbohydrates were selected (table 1 ), all related to $\beta$-glucan polysaccharide. The quality scores were between 3 and 4 according to the JADAD scale. Subjects were between 26 and 75 years, with a sample size of 18 and 97 participants $[8,18]$. Cugnet-Anceau et al. [8] evaluated the intake of $3.5 \mathrm{~g} /$ day of $\beta$-glucans in soup, in T2D patients (BMI of $20-35 \mathrm{~kg} / \mathrm{m}^{2}$ ) for 2 months. Furthermore, Maki et al. [18] assessed the effects of consuming oat $\beta$-glucans between 2.1 and 3.2 $\mathrm{g} /$ day for breakfast in hypertensive patients during 3 months. Keogh et al. [19] assessed supplements of $\beta$-glucan incorporated daily into snacks and meals in mildly hypercholesterolemic men. The dose of both treatment and control was scaled to body size for all subjects (8.1-11.9 g/day of $\beta$-glucan). The main outcomes of these interventions were $\mathrm{BP}$, lipids profile, $\mathrm{CHO}$ metabolism, and oxidative stress biomarkers. Cugnet-Anceau et al. [8] observed a decrease in triacylglycerols (TAG); besides, Maki et al. [18] demonstrated a decrease of mean peak insulin and of incremental area under the insulin curve in the $\beta$-glucans group, and an improvement of $\mathrm{BP}$ in $\mathrm{BMI}>31.5 \mathrm{~kg} / \mathrm{m}^{2} \mathrm{~T} 2 \mathrm{D}$ patients. However, any significant effect was reported in mildly hypercholesterolemic men by Keogh et al. [19].

\section{Active Lipids (CLA)}

Four articles referenced to CLAs were selected (table 1). The quality scores obtained varied from 3 to 5 within the JADAD scale. Only one of those studies was conducted among healthy subjects, with being male as the inclusion criterion (19-35 years) [20]. The subjects recruited in the other two studies were BMI $>25 \mathrm{~kg} / \mathrm{m}^{2}$, one of them with LDL phenotype B as requirement for participating, both between 35 and 65 years [21, 22]. One article assessed CLA's effect in active rheumatoid arthritis patients [23]. Sample sizes were from 40 to 92 . The administered doses ranged from $2-5.5 \mathrm{~g} /$ day in butter or capsules as oil, during periods from 5 weeks to 3 months. The main outcomes in these studies were related to BP, lipids profile, $\mathrm{CHO}$ metabolism, endothelial function, inflammatory and oxidative stress biomarkers, as well as 


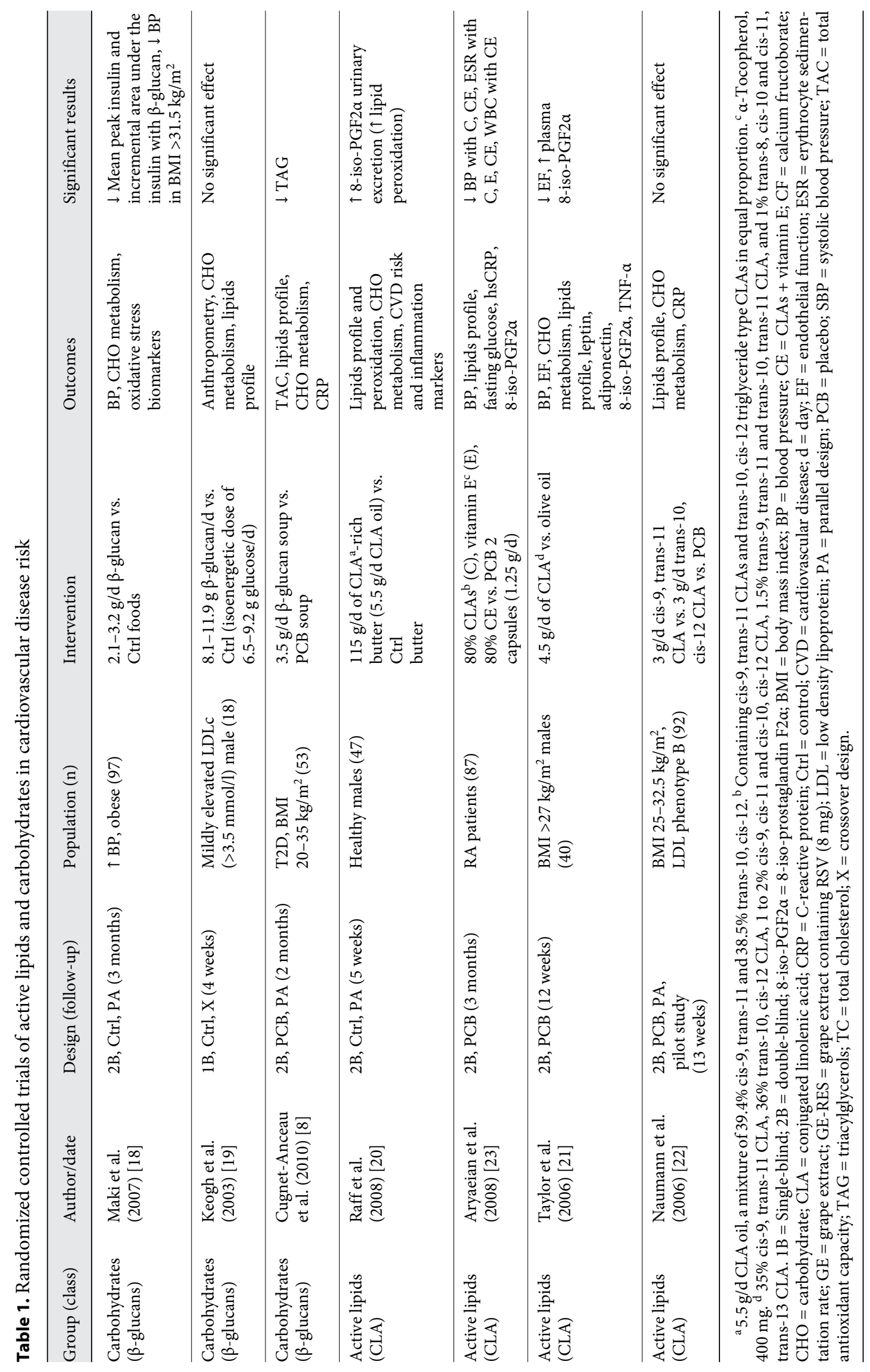




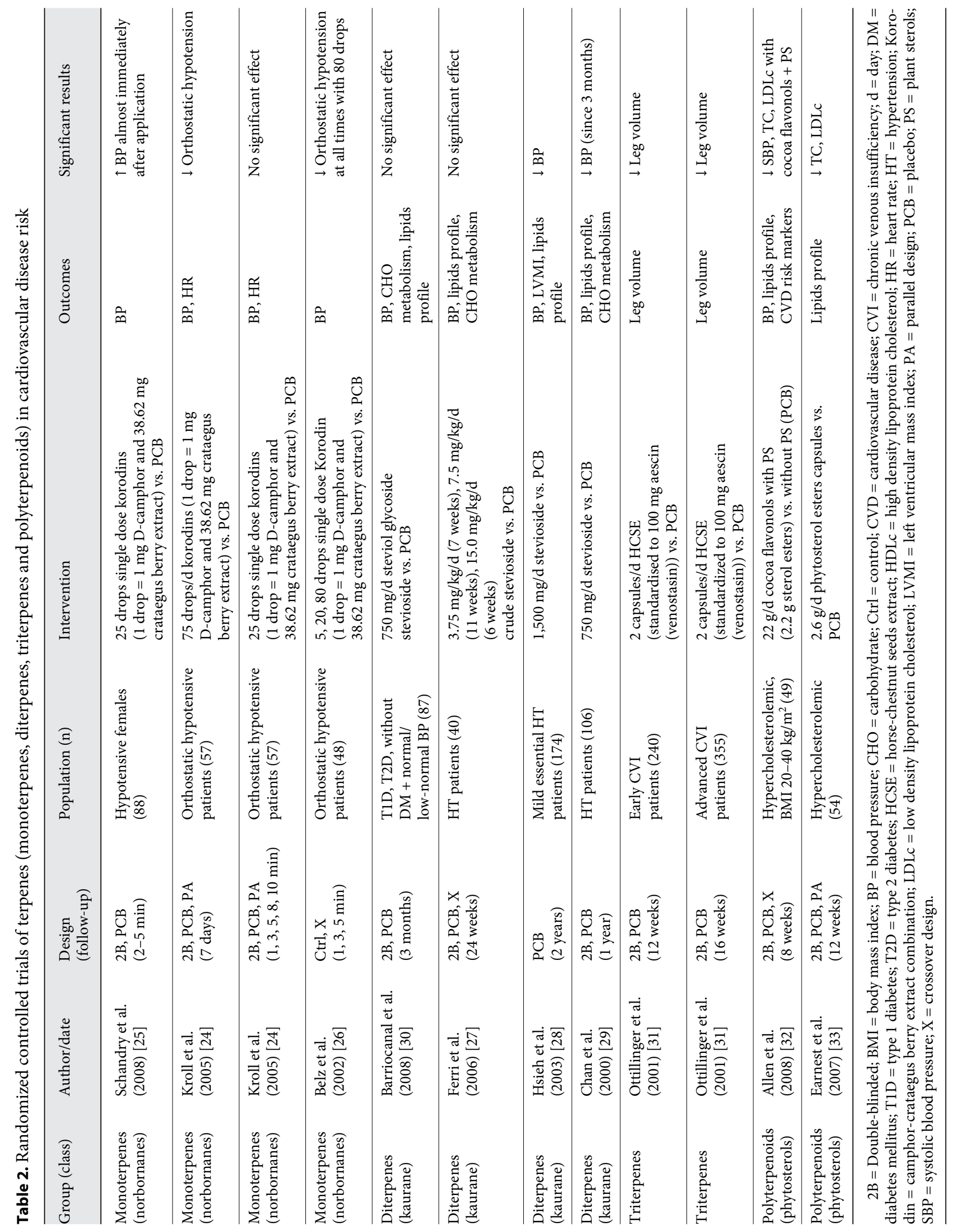


leptin, adiponectin. Naumann et al. [22] did not observe significant changes in any parameters analyzed (table 1). Moreover, there were no significant changes in plasma lipids profile. In addition, Taylor et al. [21] observed an impairment of endothelial function and increase in markers of oxidative stress, since plasma 8-iso-PGF2a increased after $4.5 \mathrm{~g} /$ day of CLA administration. In addition, Raff et al. [20] observed higher urinary excretion of 8-iso-PGF2 $\alpha$, increasing lipids peroxidation. Regarding other parameters, only BP decreased with $2 \mathrm{mg}$ /day CLAs supplementation, along with erythrocyte sedimentation rate (ESR) when CLAs were administrated in conjunction with vitamin E (400 mg/day) [23].

\section{Monoterpenes (Nobornanes)}

Three articles were reviewed and are included in table 2 . Quality score allocated according to the JADAD scale was around 3-5. Only one intervention was long-term intervention, whereas two of them focused on acute intervention. The main outcomes of the interventions regarding norbornanes were BP and HR (table 2). One study developed two interventions with 57 orthostatic hypotensive patients $>50$ years [24]: 75 drops/day Korodins (Herz-Kreislauf-Tropfen ${ }^{\circledR}$ ) for 7 days, and a single dose of 25 Korodins drops evaluated at 1, 3, 5, 8 and $10 \mathrm{~min}$. Significant changes were observed in the first intervention; thus, orthostatic hypotensive decreased. All other studies were a single dose of 25 Korodins drops in hypotensive women [25] and a single dose of 5, 20, 80 Korodin drops in orthostatic hypotensive patients (18-40 years) in both sex [26], measured at 1-5 min (1 drop = $1 \mathrm{mg} \mathrm{D}$-camphor and $38.62 \mathrm{mg}$ crataegus berry extract). In both interventions, $\mathrm{BP}$ improved after Korodins acute administration and BP increased almost immediately after its application in hypotensive women, while orthostatic hypotension decreased at all times with 80 drops according to Belz's [26] study.

The three articles selected investigated their actions in hypotensive subjects, two of them in orthostatic hypotensive [24-26]. The combination of natural D-camphor and an extract of fresh crataegus berries (Korodin Herz-Kreislauf-Tropfen ${ }^{\circledR}$ ) with a single dose of 25 drops increased BP almost immediately after application [25]; as the same pattern repeated with a dose of 80 drops after 1,3 and 5 min [26]. After one week, 75 drops/day also increase BP [24] in hypotensive subjects.

\section{Diterpenes (Kaurane)}

Four papers were included and the quality measured according to the JADAD scale ranged between 3 and 5 scores (table 2). Almost all studies were developed in HT patients [27-29], except one in which participants suffered type 1 and type 2 diabetes with normal BP or low BP [30]. Ages varied from 20 to 75 and sample size around $40-174$ subjects. The dosage interval was $300-1,500 \mathrm{mg} /$ day of steviol glycoside stevioside from three months to two years. The main outcome in all studies was increased BP, but was significantly decreased only in two studies. Other parameters when analyzed (lipids profile, $\mathrm{CHO}$ metabolism, as well as left ventricular mass index (LVMI)) did not show significant changes.

\section{Triterpenes}

One trial was chosen (table 2) and evaluated with a score of 3 according to the JADAD scale. Ottillinger and Greeske [31] separated the trial into two interventions. Both assessed the effect of 2 capsules/day HCSE (horsechestnut seeds extract) (standardized to $100 \mathrm{mg}$ aescin $\left(\right.$ Venostasin $\left.{ }^{\circledR}\right)$ ), in leg volume among 240 early chronic venous insufficiency (CVI) and 355 advanced CVI patients, for 12 and 16 weeks, respectively. The treatment caused a significant decline in leg volume in both interventions.

\section{Polyterpenoids (Phytosterols)}

Two RCTs related to polyterpenoids were finally selected (table 2), since its score as per JADAD level was from 3 to 4 . The trials were based on 17-104 volunteers in an age range 20-70. Allen et al. [32] carried out an intervention of $22 \mathrm{~g} /$ day cocoa flavanols with plant sterols (2.2 g sterol esters) provided with dark chocolate bars in hypercholesterolemic patients for a period of 8 weeks. Earnest et al. [33] examined the efficacy of ingesting $2.6 \mathrm{~g} /$ day of encapsulated phytosterol esters in hypercholesterolemic subjects for 12 weeks. Lipids profile was considered a major outcome by the two investigations. Among them, TC and LDLc decreased in both cases. With regard to BP, only Allen et al. [32] considered this aspect, resulting in the reduction of SBP. Concerning CVD risk biomarkers, changes were not observed.

\section{Nitrogen Compounds}

\section{Caffeine}

Three articles were finally selected according to our criteria (table 3), as well as based on the JADAD scale, whose score varied between 3 and 4. Lane et al. [34] investigated the effects on 47 healthy habitual coffee drinkers of $500 \mathrm{mg} /$ day of caffeine on ambulatory BP and HR for three days. After three days of caffeine administration, 


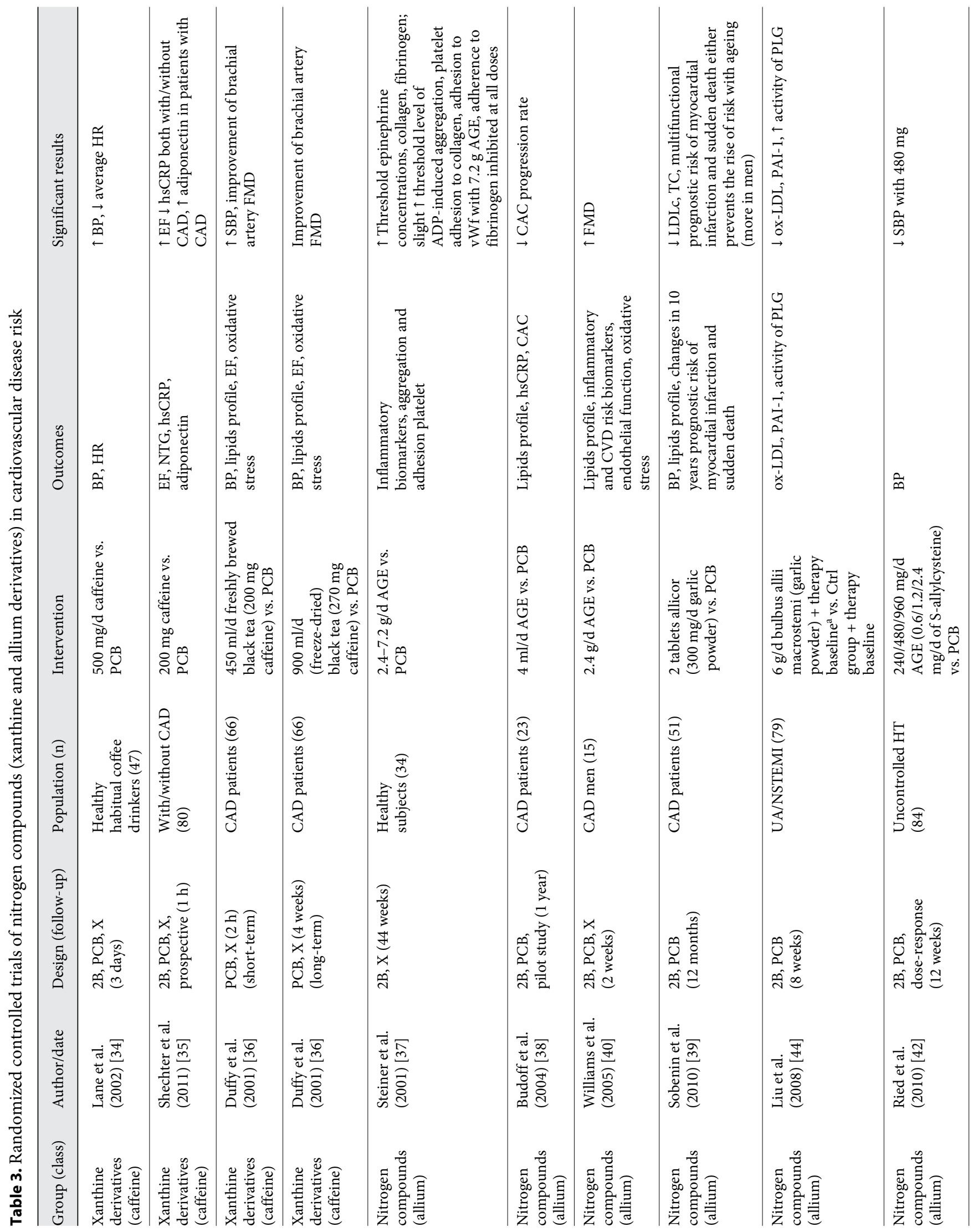




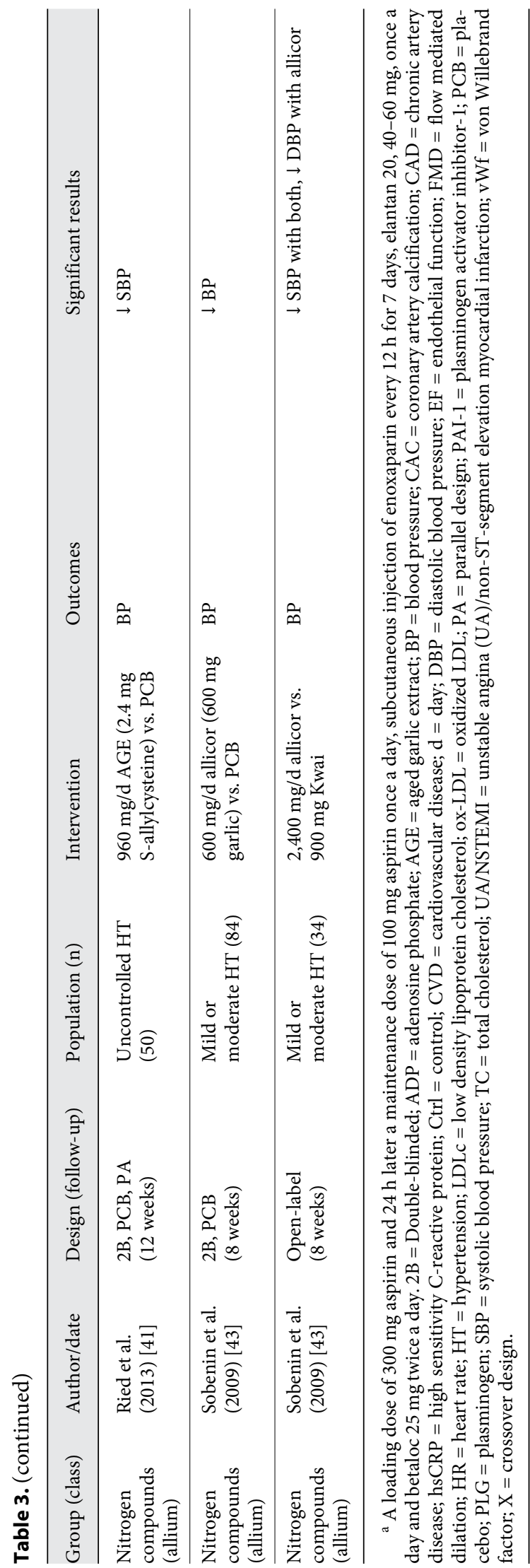

BP increased, but average HR was found to be decreased. Furthermore, Shechter et al. [35] assessed the effect of acute caffeine ingestion $(200 \mathrm{mg})$ on endothelial function, nitro-glycerine-mediated dilation (NTG) and high sensitivity hsCRP, in 80 subjects with/without coronary artery disease (CAD) [35] after one hour of administration. The endothelial function was increased; also, hsCRP decreased both in with/without CAD patients. Duffy et al. [36] also evaluated one dose of caffeine (200 $\mathrm{mg})$ in CAD patients and SBP increased after 2 hours. Moreover, the same subjects took $270 \mathrm{mg}$ caffeine contained in black tea daily for 4 weeks. In both interventions, an improvement in the endothelial function was observed, but without significant effects in relation to other parameters analyzed (table 3).

\section{Allium}

Eight articles were definitely selected due to its score in JADAD scale, hovering between the values 4 and 5 (table 3). The ages of participants were between 35 and 82 and sample size ranged from $15-84$ volunteers. One intervention was developed in 34 healthy subjects, where the efficacy of Aged Garlic Extract (AGE) was assessed at doses of 2.4-7.2 g/day for 44 weeks [37]. The main outcomes were inflammatory biomarkers, aggregation and adhesion platelet. The highest AGE supplementation increased threshold concentrations for epinephrine and collagen, fibrinogen and increased slightly threshold level of adenosine diphosphate-induced aggregation, platelet adhesion to collagen, adhesion to von Willebrand factor. Moreover, adherence to fibrinogen was potently inhibited at all levels of supplementation. Three clinical trials were related to coronary artery disease (CAD) patients: the effect of $4 \mathrm{ml} /$ day of AGE was evaluated in 23 subjects [38], while Sobenin et al. [39] investigated the effect of allicor (garlic powder) in 51 subjects, both during a period of one year. However, Williams et al. [40] assessed $2.4 \mathrm{~g} /$ day of AGE only during two weeks. The main outcomes of these studies consisted of BP, lipids profile, endothelial function, oxidative stress, inflammatory biomarkers, and coronary artery calcification (CAC), platelet aggregation and adhesion just as changes in 10 years prognostic risk of myocardial infarction and sudden death. Within lipids profile measured by the two interventions [38-40], LDLc and TC parameters were significantly decreased after allicor treatments [39]. Reduction of CAC progression was detected in the study that assessed it [38] (table 3). Sobenin et al. [39] observed a decrease in multifunctional prognostic risk of myocardial infarction and 
sudden death prevents the rise of risk with aging. Williams et al. [40] only reported significant changes in the endothelial function. Ried et al. [41] also assessed the effect of AGE in capsules $(960 \mathrm{mg} /$ day containing $2.4 \mathrm{mg}$ S-allylcysteine), as well as of different doses $(240 / 480 / 960 \mathrm{mg} /$ day $(0.6 / 1.2 / 2.4 \mathrm{mg} /$ day of S-allylcysteine)) [42] as an adjuvant to antihypertensive medication in patients with uncontrolled hypertension, both during 12 weeks. Sobenin and colleagues [43] separated the trial into two interventions. The first one included treatment that consisted of $600 \mathrm{mg} /$ day of allicor $(300$ $\mathrm{mg}$ garlic), being a randomized design, whereas the other intervention was an open-label branch, where patients received higher doses of $2,400 \mathrm{mg} / \mathrm{day}$, both patients with mild or moderate HT for 8 weeks [43]. Both authors studied the efficacy in the improvement of $\mathrm{BP}$, and SBP was significantly reduced by $1.2 \mathrm{mg} /$ day of S-allylcysteine [42]. Further, the effects of Bulbus allii macrostemi were tested on UA/NSTEMI patients, in doses of $6 \mathrm{~g} /$ day plus baseline therapy for eight weeks [44]. Markers such as ox-LDL, PAI-1 and activity of PLG were significantly improved (table 3 ).

\section{Discussion}

\section{Carbohydrates}

Maki et al. [18] assessed the effects of oat $\beta$-glucans in hypertensive subjects and observed significant changes in $\mathrm{CHO}$ metabolism and $\mathrm{BP}$ (table 1), the latter being only in subjects with $\mathrm{BMI}>31.5 \mathrm{~kg} / \mathrm{m}^{2}$. Cugnet-Anceau et al. [8] observed that a $3.5 \mathrm{~g}$ dose for 2 months of $\beta$-glucans reduced TAG in T2D subjects. Nevertheless, other plasma lipids, as well as TAC, CHO metabolism and CRP were not significantly altered. They concluded that to improve the metabolic profile of $\mathrm{T} 2 \mathrm{D}$ subjects in the long term, the quantity, the food vectors and the tolerability of $\beta$-glucan products might be reevaluated. Moreover, Keogh et al. [19] reported no clinically significant improvements in lipids profile or $\mathrm{CHO}$ metabolism; thus, they concluded that there was no evidence of improvement in CVD or T2D risk in mildly hypercholesterolemic men. With regard to previous bibliography, oat $\beta$-glucans have been used in an open-label clinical trial to reduce glucose, showing that oat $\beta$-glucans lowered postprandial glycaemia [45]. These results seem to be promising, but most antecedents were found in animal models. It is possible that subjects took a low dose in both studies, but it seems that an increase in the concentration of $\beta$-glucan negatively affects the palatability. In fact, Maki et al. [18] justified greater dropout more likely related to palatability than toleration of the study products. Chen and Raymond [46] concluded that no pure $\beta$-glucan has been used until the moment and thus, it is difficult to identify which components are effective. In the present review, authors did not use a pure extract, and consequently, clinical trials should be designed taking into account this reflection. Whether there are not systematic review and/or meta-analysis related to the effect of $\beta$-glucan along with the studies selected presented here that do not show enough evidence, we cannot recommend $\beta$-glucan in CVD.

\section{Active Lipids (CLA)}

According to the results obtained to active lipids, concretely CLA, along with previous reviews [47, 48], evidence is scarce in relation of lipids profile and on inflammatory processes, as well as $\mathrm{CHO}$ metabolism. We must take into account that the background is mainly based on animals and cells studies. Recently, Brouwer et al. [47] reviewed the effect of CLA and concluded that it increases LDL cholesterol, plasma LDL/HDL and TC/ HDL ratios. However, RCTs selected following our criteria, did not show any effect with any doses, both in healthy and unhealthy subjects. Aryaeian et al. [23] concluded that a supplementation of CLAs plus vitamin E might be profitable for heart disease prevention in RA patients. Furthermore, BP decreased after 3 months of CLAs administration [23]. Even so, there are not enough preceding publications and grade of evidence for making a recommendation in these patients. Furthermore, Taylor et al. [21], reported that CLA administration in men with $\mathrm{BMI}>27 \mathrm{~kg} / \mathrm{m}^{2}$ degraded endothelial function, apart from increasing plasma 8-iso-PGF2 $\alpha$. Additionally, Raff et al. [20] found an increase in urinary excretion of 8-iso-PGF2 $\alpha$, resulting in lipid peroxidation increased a relevant risk factor for atherosclerosis. Given the controversy and scarce evidence, further investigation is required, but it is important to consider the deleterious effect that seemed to be present in lipid peroxidation [20]. Thus, we cannot recommend CLAs against CVD.

\section{Monoterpenes (Norbornanes)}

Despite these positive results, there is no meta-analysis to support its effect. Nevertheless, it could be interesting to continue testing this product in combination with other BACs in patients with hypotension, both in acute and chronic interventions. In the future, Korodin may be applied for controlling hypotension. 


\section{Diterpenes (Kaurane)}

Many reports have shown that several classes of diterpenoids exert significant cardiovascular effects in animal models [49-51]. BP decreased in two studies, while plasma lipids were not different when compared to the placebo group in any case. In the two trials with any significant change, Kaurane was assessed for short-term [27, 30]. Although Chan et al. [29], found changes since the third month evaluating the same doses than Barriocanal et al. [30], the sample size and/or subjects' characteristics may be relevant. Only one intervention considered LVMI, from which changes were not found after intervention [28]. Because of all this, without evidence in relation to Kaurane effects in humans, recommendations may not be valid, requiring more evidence and solid results.

\section{Triterpenes}

Leg volume was the main outcome in the two interventions that were part of study, as visible sign of CVI. In both, leg volume decreased after oral HCSE in early and advanced CVI patients. We can compare such results with antecedents, since in 2012, Pittler et al. [11] published in the Cochrane Library a systematic review; its aim was to assess the evidence for HCSE as treatment of CVI with similar results in regards to reduction of mean leg volume. The adverse events reported in the reviewed trials were mild and infrequent. Thus, authors suggested that HCSE could be an effective short-term treatment option for CVI. Hence, this herbal extract may be recommended, but more rigorous RCTs are required, especially for the long term.

\section{Polyterpenoids (Phytosterols)}

Both studies selected in this review, evaluated lipid profile changes after plant sterols administration (table 2), with positive results $[32,33]$. Moreover, BP improved in the study that analyzed it [32]. Furthermore, two meta-analyses evaluated the effect of plant sterols and stanols, the first versus policosanol in hyperlipidaemia [52], concluding that the last one is a valuable addition to the usual therapeutic lifestyle changes and may be an attractive alternative to conventional lipid-lowering drugs, due to offers advantages of greater efficacy. The second study tried to minimize the potential bias due to uncontrolled confounders for guaranteeing that the calculated mean differences do not provide unbiased estimates of the effect of sterols on CVD [53]. Taking into account the rigorous analysis and their conclusions, together with our scarce evidence, sterol plants do not appear to have efficacy as a source to lower CVD risk.

\section{Nitrogen Compounds}

Caffeine

Lane et al. [34] tested $500 \mathrm{mg} /$ day of caffeine vs. placebo control for three days. Healthy, non-smoking, habitual coffee drinkers' subjects recruited and authors observed caffeine significantly raised average ambulatory $\mathrm{BP}$ during the workday and evening and reduced average HR. These results suggested that caffeine consumption may exaggerate the deleterious effects of stress in daily life and aggravate the damage to health that stress can cause. These results also demonstrated that this modest caffeine dose produced an amplification of the effects of stress on $\mathrm{BP}$ and HR during the day. The possible relation between caffeine and cardiac events is well known. Moreover, SBP also increased after two hour of caffeine administration in CAD patients in a trial carried out by Duffy et al. [36]. Particularly, BP is a strong risk factor for heart failure. Experimental studies have consistently shown that coffee and caffeine are associated with acutely raised BP [54]. Sixteen randomized controlled trials were collected in a meta-analysis, comprising 1,010 subjects, yielded an overall BP increase of 2.0/0.7 $\mathrm{mm} \mathrm{Hg}$ for regular coffee or caffeine intake. Nevertheless, it is very important to note that 9 out of 11 coffee trials were not blinded and this may have influenced the observed effects on BP. In contrast, two studies enrolled by our criteria, were double blind, as absolute requirement (according to JADAD scale), since caffeine was administrated in capsules as placebo did. Shechter et al. [35], demonstrated that $200 \mathrm{mg}$ of caffeine administered as a single dose improved brachial endothelial function in controls and patients with CAD. Duffy et al. [36] also observed an improvement in the endothelial function with the same dose and kind of patients, both after two hours and four weeks. Additionally, increased serum caffeine was associated with a decrease of inflammatory markers such as hsCRP in both with/without $\mathrm{CAD}$, and an increase in serum adiponectin patients with CAD. Other meta-analyses indicate that there is a Jshaped relationship between coffee consumption and heart failure incidence, with a modest inverse association with moderate consumption [55]. Compared with no consumption, the strongest inverse association was seen for 4 servings/day and a potentially higher risk at higher levels of consumption. It is clear that caffeine should be administered in purified capsules to prevent influence from other ingredients. In light of these findings, further evidence is required for recommending clearly caffeine administrated in capsules as moderate protection against heart failure incidence; this also depends on the kind of patients. 


\section{Allium}

Studies in animal models suggest that garlic has potential antilipidemic, antihypertensive, anti-glycaemic, antithrombotic and anti-atherogenic properties [56]. Nevertheless, evidence is scarce in relation to clinical trials. The seven trials collected in our review offered different outcomes. Gallic acid decreased BP, especially SBP in hypertensive patients [41-43]. Nevertheless, BP was also measured in CAD patients without hypertension and changes were not observed [39]. Ried et al. [57] developed a systematic review; and 11 articles were suitable for meta-analysis. Such rigorous bibliography review concluded that garlic preparations are superior to placebo in reducing BP in individuals with hypertension. Additionally, another meta-analysis developed by Reinhart et al. [58] associated gallic acid with BP reductions in patients with an elevated SBP although not in those without elevated SBP. Using the SIGN guidelines, both works obtained $1+$ by level of evidence. Consequently, we could establish recommendations for allium as far as effect on BP in hypertensive subjects. On the other hand, both authors emphasize that it is required to investigate the effect in long-term trials. Stabler et al. [59] concluded that garlic reduces mean supine BP versus placebo. However, they identified that the confidence intervals for these effect estimates are not precise by means of metaanalysis; thus, there is difficulty in recommending its use. We should take into account that Stabler et al. [59] studies, were valuated with $1+$ and they did not suggest the recommendation of allium due to insufficient evidence. Simons et al. [60] evaluated 32 studies by meta-analyses and from their critical point of view, the methodological quality of the studies was poor. The meta-analysis was very rigorous, obtaining $1++$ score according to SIGN guidelines and level of evidence is considered high. In their view, the use of garlic cannot be recommended as antihypertensive advice for hypertensive patients in daily practice [60].

With regard to the lipids profile, a decrease in TC and LDLc after [61] consuming garlic powder [39] for one year was observed in CAD patients. Although, $4 \mathrm{ml} /$ day of AGE not caused any changes in plasma lipids, only in CAC progression rate [38]. Zeng et al. [62] elaborated a meta-analysis and concluded that garlic was superior to placebo in reducing TC and TAG concentrations and concluded that garlic therapy should benefit patients with risk of CAD according. Nevertheless, other serum lipid parameters such as LDLc, HDLc, Apo-B and TC/HDLc were not significantly affected. The level of evidence through SIGN guidelines resulted in $1++$, so that allium

Bioactive Compounds in Cardiovascular Disease could be recommended in subjects with cardiovascular risk with a grade of recommendation of A. Based on these facts, allium (gallic acid, especially) is beneficial for CAD prevention. Furthermore, there is a great quality metaanalysis focused on BP and characterized by its rigorous methodology [60]. Additionally, Sobenin et al. [39] also observed a decrease of multifunctional prognostic risk of myocardial infarction and sudden death prevents the rise of risk with aging; the most significant effects were observed in men. We must also take into account the decrease of the CAC progression rate [38], which is an excellent marker of overall coronary atherosclerotic burden correlating with both the total plaque burden and the presence of obstructive disease [63]. With reference to Williams et al. [40], we must consider that the evaluation of AGE was only during two weeks, and this period is short to consider the changes. Even so, endothelial function improved after such intervention.

We must mention the changes in UA/NSTEMI using Bulbus allii macrostemi, which decreased ox-LDL, PAI-1, apart from to increase the activity of plasminogen [44]. Results are promising in relation to Allium sativum in the CVD prevention, but further evidence in long-term is needed for verifying such facts, especially Bulbus allii macrostemi alone, without baseline therapy.

Certain limitations need to be considered. First, $\mathrm{MeSH}$ terms are not often used by researchers. Such specific terms must be taken into account when articles are drafted, assuring a good indexation and more visibility, facilitating the evidence valuation. Second, the application of resources such as CONSORT (Consolidated Standards of Reporting Trials) statement or JADAD scale is highly scarce. Moreover, the existence of checklists helps authors and editors to improve the reporting of RCTs and consequently provides scientific quality in data report. We consider that a clinical trial is reliable when it is at least randomized and blinded. Then, trials included in this review have a high level of heterogeneity, making it more difficult to draw concrete conclusions in relation to the kind of subjects, format of analyzed product or its combination with other compounds.

\section{Conclusions}

The role of BACs as adjuvants in CVD is increasing and validation of its effects is essential. Evidence shows that BAC such as horse chestnut seed extract, sterol plants and allium derivatives are helpful in decreasing risk factors of CVD. However, it is necessary to develop 
better quality RCTs (crossover design, double-blinded, long term, placebo/controlled) and furthermore, elaborate rigorous meta-analysis of existence evidence to support the effect of BAC on prevention and treatment of CVD.

\section{Acknowledgments}

The authors are grateful for the support of Mrs. Mercedes Rodriguez del Castillo Martin in developing some technical assistances with the search in databases.

\section{Author Contributions}

B.P.-V., O.D.R.-H., C.M.A., A.G. contributed to the planning of the search of the literature, designed the analysis and results presentation and created the tool for assessing the quality of the articles. B.P.-V., O.D.R.-H. were involved in the analyses of the articles. B.P.-V., O.D.R.-H. wrote the draft. All authors discussed and revised all drafts and approved the final manuscript.

\section{Disclosure Statement}

There are no conflicts of interest to report.

\section{References}

1 Martínez-Augustin O, Aguilera CM, Gil- 12 Law MR: Plant sterol and stanol margarines Campos M, Sánchez de Medina F, Gil A: Bioactive anti-obesity food components. Int J Vitam Nutr Res 2012;82:148-156.

2 World Health Organization: Obesity and Overweight, 2015.

3 Perk J, De Backer G, Gohlke H, Graham I, Reiner Z, Verschuren M, et al: European Guidelines on cardiovascular disease prevention in clinical practice (version 2012). Eur Heart J 2012;33:1635-1701.

-4 Stone NJ, Robinson JG, Lichtenstein AH, Bairey Merz CN, Blum CB, Eckel RH, et al: 2013 ACC/AHA guideline on the treatment of blood cholesterol to reduce atherosclerotic cardiovascular risk in adults: a report of the American college of cardiology/American heart association task force on practice guidelines. J Am Coll Cardiol 2014;63:2889-2934.

5 Kitts DD: Bioactive substances in food: identification and potential uses. Can J Physiol Pharmacol 1994;72:423-434.

6 Kris-Etherton PM, Hecker KD, Bonanome A, Coval SM, Binkoski AE, Hilpert KF, et al: Bioactive compounds in foods: their role in the prevention of cardiovascular disease and cancer. Am J Med 2002;113(suppl 9B):71S-88S.

7 Food and Drug Administration: FDA Basics - Dietary Supplements, 2010.

$\checkmark 8$ Cugnet-Anceau C, Nazare JA, Biorklund M, Le Coquil E, Sassolas A, Sothier M, et al: A controlled study of consumption of beta-glucan-enriched soups for 2 months by type 2 diabetic free-living subjects. Br J Nutr 2010; 103:422-428.

$\checkmark 9$ Chinnadurai K, Kanwal HK, Tyagi AK, Stanton C, Ross P: High conjugated linoleic acid enriched ghee (clarified butter) increases the antioxidant and antiatherogenic potency in female Wistar rats. Lipids Health Dis 2013;12:121.

10 Crowell PL: Prevention and therapy of cancer by dietary monoterpenes. J Nutr 1999;129: 775S-778S

11 Pittler MH, Ernst E: Horse chestnut seed extract for chronic venous insufficiency. Cochrane Database Syst Rev 2012;11:CD003230. and health. West J Med 2000;173:43-47.

13 Thompson GR, Grundy SM: History and development of plant sterol and stanol esters for cholesterol-lowering purposes. Am J Cardiol 2005;96:3-9.

14 Rahman K, Lowe GM: Garlic and cardiovascular disease: a critical review. J Nutr 2006; 136(3 suppl): 736 S-740S.

15 Scottish Intercollegiate Guidelines Network: Risk Estimation and the Prevention of Cardiovascular Disease: A National Clinical Guideline (97), 2007. http://www.sign.ac.uk/ pdf/sign97.pdf.

16 Jadad AR, Moore RA, Carroll D, Jenkinson C, Reynolds DJ, Gavaghan DJ, et al: Assessing the quality of reports of randomized clinical trials: is blinding necessary? Control Clin Trials $1996 ; 17: 1-12$

17 Uauy R: Fats and Fatty Acids in Human Nutrition: Report of An Expert Consultation. FAO Food and Nutrition Paper, 2008, vol 550, p 189

18 Maki KC, Galant R, Samuel P, Tesser J, Witchger MS, Ribaya-Mercado JD, et al: Effects of consuming foods containing oat betaglucan on blood pressure, carbohydrate metabolism and biomarkers of oxidative stress in men and women with elevated blood pressure. Eur J Clin Nutr 2007;61:786-795.

19 Keogh GF, Cooper GJ, Mulvey TB, McArdle $\mathrm{BH}$, Coles GD, Monro JA, et al: Randomized controlled crossover study of the effect of a highly beta-glucan-enriched barley on cardiovascular disease risk factors in mildly hypercholesterolemic men. Am J Clin Nutr 2003;78:711-718

20 Raff M, Tholstrup T, Basu S, Nonboe P, Sørensen MT, Straarup EM: A diet rich in conjugated linoleic acid and butter increases lipid peroxidation but does not affect atherosclerotic, inflammatory, or diabetic risk markers in healthy young men. J Nutr 2008;138:509-514. 21 Taylor JS, Williams SR, Rhys R, James P, Frenneaux MP: Conjugated linoleic acid impairs endothelial function. Arterioscler Thromb Vasc Biol 2006;26:307-312.
22 Naumann E, Carpentier YA, Saebo A, Lassel TS, Chardigny JM, Sébédio JL, et al: Cis-9, trans-11 and trans-10, cis-12 conjugated linoleic acid (CLA) do not affect the plasma lipoprotein profile in moderately overweight subjects with LDL phenotype B. Atherosclerosis 2006;188:167-174.

23 Aryaeian N, Shahram F, Djalali M, Eshragian MR, Djazayeri A, Sarrafnejad A, et al: Effect of conjugated linoleic acid, vitamin $\mathrm{E}$ and their combination on lipid profiles and blood pressure of Iranian adults with active rheumatoid arthritis. Vasc Health Risk Manag 2008; 4:1423-1432.

24 Kroll M, Ring C, Gaus W, Hempel B: A randomized trial of Korodin Herz-KreislaufTropfen as add-on treatment in older patients with orthostatic hypotension. Phytomedicine 2005; 12:395-402.

25 Schandry R, Duschek S: The effect of Camphor-Crataegus berry extract combination on blood pressure and mental functions in chronic hypotension - a randomized placebo controlled double blind design. Phytomedicine 2008; 15:914-922.

26 Belz GG, Butzer R, Gaus W, Loew D: Camphor-Crataegus berry extract combination dose-dependently reduces tilt induced fall in blood pressure in orthostatic hypotension. Phytomedicine 2002;9:581-588.

27 Ferri LA, Alves-Do-Prado W, Yamada SS, Gazola S, Batista MR, Bazotte RB: Investigation of the antihypertensive effect of oral crude stevioside in patients with mild essential hypertension. Phytother Res 2006;20:732-736.

28 Hsieh MH, Chan P, Sue YM, Liu JC, Liang TH, Huang TY, et al: Efficacy and tolerability of oral stevioside in patients with mild essential hypertension: a two-year, randomized, placebo-controlled study. Clin Ther 2003;25: 2797-2808.

29 Chan P, Tomlinson B, Chen YJ, Liu JC, Hsieh $\mathrm{MH}$, Cheng JT: A double-blind placebo-controlled study of the effectiveness and tolerability of oral stevioside in human hypertension. Br J Clin Pharmacol 2000;50:215-220. 
-30 Barriocanal LA, Palacios M, Benitez G, Benitez S, Jimenez JT, Jimenez N, et al: Apparent lack of pharmacological effect of steviol glycosides used as sweeteners in humans. A pilot study of repeated exposures in some normotensive and hypotensive individuals and in type 1 and type 2 diabetics. Regul Toxicol Pharmacol 2008;51:37-41.

-31 Ottillinger B, Greeske K: Rational therapy of chronic venous insufficiency - chances and limits of the therapeutic use of horse-chestnut seeds extract. BMC Cardiovasc Disord 2001; $1: 5$.

- 32 Allen RR, Carson L, Kwik-Uribe C, Evans EM, Erdman JW Jr: Daily consumption of a dark chocolate containing flavanols and added sterol esters affects cardiovascular risk factors in a normotensive population with elevated cholesterol. J Nutr 2008;138:725-731.

-33 Earnest CP, Mikus CR, Lemieux I, Arsenault BJ, Church TS: Examination of encapsulated phytosterol ester supplementation on lipid indices associated with cardiovascular disease. Nutrition 2007;23:625-633.

-34 Lane JD, Pieper CF, Phillips-Bute BG, Bryant JE, Kuhn CM: Caffeine affects cardiovascular and neuroendocrine activation at work and home. Psychosom Med 2002;64:595-603.

- 35 Shechter M, Shalmon G, Scheinowitz M, Koren-Morag N, Feinberg MS, Harats D, et al: Impact of acute caffeine ingestion on endothelial function in subjects with and without coronary artery disease. Am J Cardiol 2011; 107:1255-1261.

-36 Duffy SJ, Keaney JF Jr, Holbrook M, Gokce N, Swerdloff PL, Frei B, et al: Short- and longterm black tea consumption reverses endothelial dysfunction in patients with coronary artery disease. Circulation 2001;104:151-156.

-37 Steiner M, Li W: Aged garlic extract, a modulator of cardiovascular risk factors: a dosefinding study on the effects of AGE on platelet functions. J Nutr 2001;131:980S-984S.

- 38 Budoff MJ, Takasu J, Flores FR, Niihara Y, Lu $\mathrm{B}$, Lau BH, et al: Inhibiting progression of coronary calcification using aged garlic extract in patients receiving statin therapy: a preliminary study. Prev Med 2004;39:985-991.

-39 Sobenin IA, Pryanishnikov VV, Kunnova LM, Rabinovich YA, Martirosyan DM, Orekhov AN: The effects of time-released garlic powder tablets on multifunctional cardiovascular risk in patients with coronary artery disease. Lipids Health Dis 2010;9:119.

40 Williams MJ, Sutherland WH, McCormick MP, Yeoman DJ, de Jong SA: Aged garlic extract improves endothelial function in men with coronary artery disease. Phytother Res 2005;19:314-319.
41 Ried K, Frank OR, Stocks NP: Aged garlic extract reduces blood pressure in hypertensives: a dose-response trial. Eur J Clin Nutr 2013;67: 64-70.

42 Ried K, Frank OR, Stocks NP: Aged garlic extract lowers blood pressure in patients with treated but uncontrolled hypertension: a randomised controlled trial. Maturitas 2010;67: 144-150.

43 Sobenin IA, Andrianova IV, Fomchenkov IV, Gorchakova TV, Orekhov AN: Time-released garlic powder tablets lower systolic and diastolic blood pressure in men with mild and moderate arterial hypertension. Hypertens Res 2009;32:433-437.

44 Liu Y, Zhang L, Liu YF, Yan FF, Zhao YX: Effects of Bulbus allii macrostemi on clinical outcomes and oxidized low-density lipoprotein and plasminogen in unstable angina/ non-ST-segment elevation myocardial infarction patients. Phytother Res 2008;22: 1539-1543.

-45 Jenkins AL, Jenkins DJ, Zdravkovic U, Würsch P, Vuksan V: Depression of the glycemic index by high levels of beta-glucan fiber in two functional foods tested in type 2 diabetes. Eur J Clin Nutr 2002;56:622-628.

46 Chen J, Raymond K: Beta-glucans in the treatment of diabetes and associated cardiovascular risks. Vasc Health Risk Manag 2008;4: 1265-1272.

47 Brouwer IA, Wanders AJ, Katan MB: Trans fatty acids and cardiovascular health: research completed? Eur J Clin Nutr 2013;67:541-547.

48 Aminot-Gilchrist DV, Anderson HD: Insulin resistance-associated cardiovascular disease: potential benefits of conjugated linoleic acid. Am J Clin Nutr 2004;79(6 suppl):1159S$1163 \mathrm{~S}$.

- 49 Tirapelli CR, Ambrosio SR, da Costa FB, Coutinho ST, de Oliveira DC, de Oliveira AM: Analysis of the mechanisms underlying the vasorelaxant action of kaurenoic acid in the isolated rat aorta. Eur J Pharmacol 2004;492: 233-241.

50 Tirapelli CR, Ambrosio SR, de Oliveira AM, Tostes RC: Hypotensive action of naturally occurring diterpenes: a therapeutic promise for the treatment of hypertension. Fitoterapia 2010;81:690-702

51 Hipólito UV, Rodrigues GJ, Lunardi CN, Bonaventura D, Ambrosio SR, de Oliveira AM, et al: Mechanisms underlying the vasorelaxant action of the pimarane ent-8(14),15-pimaradien-3beta-ol in the isolated rat aorta. Eur J Pharmacol 2009;616:183-191.

52 Chen JT, Wesley R, Shamburek RD, Pucino F, Csako G: Meta-analysis of natural therapies for hyperlipidemia: plant sterols and stanols versus policosanol. Pharmacotherapy 2005; 25:171-183.

53 Genser B, Silbernagel G, De Backer G, Bruckert E, Carmena R, Chapman MJ, et al: Plant sterols and cardiovascular disease: a systematic review and meta-analysis. Eur Heart J 2012;33:444-451.

- 54 Noordzij M, Uiterwaal CS, Arends LR, Kok FJ, Grobbee DE, Geleijnse JM: Blood pressure response to chronic intake of coffee and caffeine: a meta-analysis of randomized controlled trials. J Hypertens 2005;23:921-928.

55 Mostofsky E, Rice MS, Levitan EB, Mittleman MA: Habitual coffee consumption and risk of heart failure: a dose-response meta-analysis. Circ Heart Fail 2012;5:401-405.

56 Kim-Park S, Ku DD: Garlic elicits a nitric oxide-dependent relaxation and inhibits hypoxic pulmonary vasoconstriction in rats. Clin Exp Pharmacol Physiol 2000;27:780-786.

57 Ried K, Frank OR, Stocks NP, Fakler P, Sullivan T: Effect of garlic on blood pressure: a systematic review and meta-analysis. BMC Cardiovasc Disord 2008;8:13.

58 Reinhart KM, Coleman CI, Teevan C, Vachhani P, White CM: Effects of garlic on blood pressure in patients with and without systolic hypertension: a meta-analysis. Ann Pharmacother 2008;42:1766-1771.

59 Stabler SN, Tejani AM, Huynh F, Fowkes C: Garlic for the prevention of cardiovascular morbidity and mortality in hypertensive patients. Cochrane Database Syst Rev 2012; 8:CD007653.

60 Simons $\mathrm{S}$, Wollersheim $\mathrm{H}$, Thien T: A systematic review on the influence of trial quality on the effect of garlic on blood pressure. Neth J Med 2009;67:212-219.

-61 Budoff MJ, Ahmadi N, Gul KM, Liu ST, Flores FR, Tiano J, et al: Aged garlic extract supplemented with B vitamins, folic acid and L-arginine retards the progression of subclinical atherosclerosis: a randomized clinical trial. Prev Med 2009;49:101-107.

62 Zeng T, Guo FF, Zhang CL, Song FY, Zhao $\mathrm{XL}$, Xie KQ: A meta-analysis of randomized, double-blind, placebo-controlled trials for the effects of garlic on serum lipid profiles. J Sci Food Agric 2012;92:1892-1902.

- 63 Budoff MJ, Achenbach S, Blumenthal RS, Carr JJ, Goldin JG, Greenland P, et al: Assessment of coronary artery disease by cardiac computed tomography: a scientific statement from the American heart association committee on cardiovascular imaging and intervention, council on cardiovascular radiology and intervention, and committee on cardiac imaging, council on clinical cardiology. Circulation 2006;114:1761-1791. 